\title{
A clinical interpretation of shame regulation in maladaptive perfectionism
}

Frans Schalkwijk $^{\mathrm{c}, *}$, Eus J.W. Van Someren ${ }^{\mathrm{a}, \mathrm{b}}$, Rick Wassing ${ }^{\mathrm{a}}$

a Dep. Sleep and Cognition, Netherlands Institute for Neuroscience, Meibergdreef 47, 1105

BA Amsterdam, the Netherlands ${ }^{b}$ Dep. Integrative Neurophysiology, Neuroscience Campus, VU University and Medical Center, De Boelelaan 1085, 1081 HV Amsterdam, the Netherlands ${ }^{c}$ Dep. of Education, Program Group Forensic Child and Youth Care, the Netherlands

\begin{abstract}
Maladaptive perfectionism, a transdiagnostic personality trait, is associated with depression. Shame has been associated with depressive disorders and to negatively impact its treatment. Interestingly, maladaptive perfec- tionism and maladaptive shame regulation strategies might be both expressions of an internalizing personality style. We applied forward and backward stepwise linear regression modeling to explain maladaptive perfectionism by association of shame coping styles in a highly powered cross-sectional study of 1436 participants.

Maladaptive perfectionism was shown to have significant positive associations with the two internalizing coping styles attacking the self, and withdrawing, and negative associations with adaptive coping. Furthermore, a significant positive interaction effect was shown for the two internalizing coping styles.

Our research shows that participants who adopt maladaptive perfectionism, internalize the pain of shame. Clinically, this is especially relevant during treatment: the patterns of maladaptive perfectionism must come into the open, the client feels exposed and, consistent with his shame regulation style, shame grows. Our research might account for the negative responsiveness of perfectionism to brief therapies for depression: there may not be enough focus on and time for working through the evoked shame.
\end{abstract}




\section{Introduction}

Perfectionism is clinically meaningful: it can be considered as a transdiagnostic personality trait, contributing to the etiology and maintenance of multiple psychiatric disorders (Egan et al., 2011). A meta-analysis of 10 longitudinal studies suggested that perfectionism dimensions are part of the premorbid personality of people vulnerable to depressive symptoms: Seven perfectionism dimensions contributed to perfectionism-depression effects in a small magnitude (Smith, Sherry, Rnic, Saklofske, Enns \& Gralnick, 2016).

Diagnostically, perfectionism is associated with OCD (Egan et al., 2011; Glickauf-Hughes and Wells, 1995), narcissism (Mann, 2004) and depression (Ashby et al., 2006; Jahromi et al., 2012; Malinowski, Veselka \& Atkinson, 2017; Smith, Saklofske, Yan \& Sherry, 2017). Perfectionism is also negatively related to e.g. responsiveness to brief therapies for depression (Blatt et al., 1998) and the quality of the therapeutic alliance (Black et al., 2013).

In the research literature, perfectionism is understood as "striving for flawlessness and setting excessively high standards for performance, accompanied by tendencies for overly critical evaluations of their behavior" (Stoeber and Yang, 2010). Most authors advocate a multidimensional model of perfectionism, which does justice to the clinical reality of its twosidedness as being potentially adaptive or maladaptive (Frost et al., 1990; Hewitt and Flett, 1991; Kim, Chen, MacCann, Karlov \& Kleitman, 2015; Sironic and Reeve, 2015; Stoeber \& Otto, 2006). The criterion for differentiating between the two is "The perception that one consistently fails to meet the high standards one has set for oneself" (Slaney et al., 2002). This dimension is called 'discrepancy', the subjectively perceived gap between one's performance standards and one's self-evaluation of adequacy. Adaptive and healthy perfectionism is associated with low discrepancy and low negligible levels of self-criticism, although personal standards for performance can be compatible to those of maladaptive perfectionism (Rice and Stuart, 2010). Maladaptive perfectionism is associated with high personal performance standards and at the same time strong criticism in self-evaluation or perceived inadequacy in meeting one's standards. These theoretical constructs have been operationalized in the three scales of the Almost Perfect Scale (APS-R): High Standards, Order and Discrepancy (Slaney et al., 2001).

Since the introduction of the APS-R, research has shown that 'High Standards' and 
'Order' measure adaptive, positive perfectionism, whereas 'Discrepancy' measures a negative attribute, associated with Five-Factor Model's (FFM) neuroticism (Rice et al., 2007). Another study, relating the APS-R to the Minnesota Multiphasic Personality Inventory (Rice and Stuart, 2010), found that subjects with high Discrepancy scores are likely to experience depression, negative emotionality, and psychological tension. High Standards with low Discrepancy-scores on the other side, were associated with high self-esteem, more positive emotions, less psychological tension, and overall better psychological health. In the same study, high Discrepancy had a strong positive association with a motivational style aiming at avoiding pain. Henceforth, we will call perfectionism with high Discrepancy scores 'maladaptive perfectionism' and perfectionism with low Discrepancy scores 'adaptive perfectionism'.

The Discrepancy score can be interpreted as a reflection of the subjectively perceived gap between performance standards and one's self-evaluation of (in)adequacy. Attribution theory learns that the subjective valence attached to the perceived gap leads to different kinds of self-conscious emotions, such as pride, shame or guilt (Mills, 2005; Schalkwijk, 2015; Stoeber et al., 2013). Now, the question comes to the fore, whether adaptive and maladaptive perfectionism is associated with different self-conscious emotions (Stoeber et al., 2007; Tangney and Dearing, 2003). Fedewa et al. (2005) found that maladaptive perfectionism correlated positively with shame and guilt proneness, whereas adaptive perfectionism demonstrated a positive association with pride proneness and negative associations to shame and anxiety proneness. This study underlined the important association between proneness to experiencing shame and guilt and maladaptive perfectionism, but it did not consider the various ways of coping with self-conscious emotions; whether the adaptive and maladaptive dimensions of perfectionism are congruent with the adaptive and maladaptive qualities that have been defined for coping with self-conscious emotions.

We are interested in the ways experienced shame is regulated by the subject: How does one cope with it? We used the Compass of Shame Scale, a six-subscale measure for shame regulation (Elison et al., 2006b; Schalkwijk et al., 2016). The two internalizing shame regulation strategies are 'attacking the self', representing inward-directed anger and selfblame, thus magnifying the emotional conflict, and 'withdrawing', representing the tendency to hide or withdraw when shamed, thus reducing the emotional experience. The two externalizing shame regulation strategies are 'avoiding', representing disavowal and emotional distancing, thus minimizing the situation in something neutral or even positive, and 'attacking the other', representing outward-directed anger and blaming others. By 
attacking others, the source of the shame is projected onto another ("You make me feel ashamed") and consequently one feels entitled to being angry with the other. So, the anger resulting from experiencing shame is still in awareness, but not attributed to the self anymore. These four shame regulation strategies are considered to be maladaptive and in contrast to 'adaptive shame regulation', which is associated with a minimum of distortion of the shame emotion: it captures the acknowledgement of shame and motivation to apologize and/or make amends. Finally, 'shame-proneness', the tendency to react in a situation with shame, is assessed.

Our first hypothesis is that maladaptive perfectionism is strongly positively associated with a regulation style that blames the discrepancy on the self ('Attack Self'). We hypothesize a weak negative association between maladaptive perfectionism on the one hand and withdrawal from shameful experiences and emotional distancing on the other hand, as these two shame regulation styles try to minimize the experience of shame by not paying too much attention to it or even denying it. Our third hypothesis is that maladaptive perfectionism is strongly negatively associated with outward-directed aggression, as in this shame regulation style the subject attributes the source of distress to others and not to the self and would also be inclined to externalize reasons for not living up to one's expectations. Maladaptive perfectionism is expected to be negatively associated with high adaptive coping: subjects that can successfully cope with shame over failures are not likely to evaluate their performance negatively. Finally, maladaptive perfectionism is hypothesized to be positively associated with the tendency to feel exposed to shame.

\section{Methods}

\subsection{Participants}

The internet surveys described below were obtained through the Netherlands Sleep Registry (Benjamins et al., 2016) from 1436 volunteers (350 males [24.4\%], 1086 females [75.6\%]). Recruitment was accomplished by adverts on our research-group's website, but also on television, radio, magazines, newspapers, and flyers distributed in healthcare centers and conventions. Participants had a mean age \pm SD of $51.3 \pm 14.4$ years. Males were significantly older compared to the females $\left(56.2 \pm 13.4\right.$ vs. $49.8 \pm 14.4$ years; $\left.t[629.80]=7.69, p<10^{-13}\right)$. Since volunteers did not have any interventions or behavioral constraints, anonymous 
participation without informed consent was approved by both the Medical Ethical Committee of the Academic Medical Center of the University of Amsterdam and the Central Committee on Research Involving Human Subjects (CCMO), The Hague, The Netherlands.

\subsection{Materials}

\section{Compass of Shame Scale}

To assess how subjects regulate shameful experiences and how prone they are to experiencing shame, we used the Dutch version of the Compass of Shame Scale (CoSS) (Schalkwijk et al., 2016). The CoSS uses four descriptive situations that could be evaluated to be shameful. For each situation, participants have to rate in ten answers how often they would actually feel and apply particular regulation strategies to deal with the resulting shame. Answer options are "never", "seldom", "sometimes", "often", and "almost always". The subscales of the CoSS are the internalizing subscales 'Attack Self' (range: 0-16) and 'Withdrawal' (range: 0-16), the externalizing subscales 'Avoidance' (range: 0-40) and 'Attack Other' (range: 0-28), the adaptive subscale 'Adaptive' (range: 0-32), and the subscale 'Shame-proneness' (range: 0-16). These subscales are not mutually exclusive, i.e. across people, various combinations of coping styles are applied.

\section{Almost Perfect Scale-Revised}

Maladaptive perfectionism was measured with the Discrepancy subscale of the Almost Perfect Scale-Revised (APS-R) (Slaney et al., 2001), which is comprised of 12 first-person statements regarding the evaluation of oneself meeting his or her perfectionism goals. Answer options are on a 7-point Likert scale ("strongly disagree", "disagree", "slightly disagree", "neutral”, “agree", "slightly agree”, and "strongly agree"; range 0-83). Low scores on this subscale are taken as adaptive perfectionism and vice versa.

\subsection{Statistical analyses}

Since the units of the used scales are arbitrary and for generalization to a normally distributed population all subscales are $z$-transformed. All hypotheses were evaluated in a single linear model. We applied forward and backward stepwise linear regression modeling in MATLAB R2016a (9.0.0.341360; The MathWorks Inc., Natick, MA, United States). Stepwise linear 
regression algorithms circumvent selection bias by iteratively searching for the independent variable to add or remove from the model based on an a priori selection criterion. The initial model was specified to include all CoSS subscales, and Sex and Age as likely confounders, $P D \sim \beta \cdot S H+\beta \cdot A D+\beta \cdot A S+\beta \cdot W D+\beta \cdot A O+\beta \cdot A V+\beta \cdot \operatorname{Sex}+\beta \cdot$ Age

where SH is Shame-proneness, AD is Adaptive, AS is Attack Self, WD is Withdrawal, AO is Attack Other, and AV is Avoidance. The largest set of terms in the fit was set to include all possible interaction effects among the terms. The term-inclusion criterion was set to increase the Adjusted- $R^{2}$ by at least 0.0025 , and the exclusion criterion was set if a term did not add to the total explained adjusted- $R^{2}$, i.e. $\Delta$ Adjusted $-R^{2}=0$.

\section{Results}

We applied forward and backward linear-regression modeling to explain (mal)adaptive perfectionism as a function of all CoSS subscales. The initial model had an Adjusted- $R^{2}$ of 0.348. The model converged after four iterations. In the first iteration an interaction term between Attack Self and Withdrawal was added to the model (Adjusted- $R^{2}=0.352$ ), and successively three terms were removed: (2) Avoidance, (3) Shame-proneness, and (4) Age. The resulting model was comprised of three significant main effects and one interaction between the subscales Attack Self and Withdrawal (Table 1). Overall, the model was highly significant $\left(F[6,1429]=131 ; p<10^{-131}\right.$; Adjusted- $\left.R^{2}=0.352\right)$. The magnitude of the effect sizes was interpreted small if $\beta<0.20$, medium if $0.20 \leq \beta<.50$, and large if $\beta \geq .50$ (Cohen, 1992).

$<$ Insert table 1 here $>$

Perfectionism Discrepancy was positively associated with high Attack Self: the more subjects blame themselves for their own shortcomings, the more they showed maladaptive perfectionism $\left(\beta=0.36 ; t[1436]=11.61 ; p<10^{-29}\right)$. Furthermore, a positive association was found for Withdrawal $\left(\beta=0.20 ; t[1436]=5.92 ; p<10^{-8}\right)$, indicating that subjects who tend to withdraw themselves from shameful situations, still blame themselves for failing in realizing their expectations. Moreover, there was a small positive interaction effect between Attack Self and Withdrawal on maladaptive perfectionism $(\beta=0.06 ; t[1436]=3.09 ; p<$ 
$.005)$, indicating that subjects who experience shame as a personal failure ánd withdraw from the shameful situation, will express maladaptive perfectionism even stronger.

Although the effect size was small, Adaptive coping was shown to negatively associate with Perfectionism Discrepancy $(\beta=-0.08 ; t[1436]=-3.49 ; p<.0005)$, whereas the association was non-significant for Attack Other $(\beta=0.04 ; t[1436]=1.61 ; p=.11)$. Shameproneness and Avoidance did not survive the model-criterion. Finally, sex was able to explain enough variance to be kept in the model, yet its association with Perfectionism Discrepancy was non-significant $(\beta=0.05 ; t[1436]=1.05 ; p=.29)$.

\section{Discussion}

Given the clinical relevancy of maladaptive perfectionism in relation towards depression (Yelsma et al., 2002) and the negative influence of shame in the short term treatment of depression (Blatt et al., 1998; Schalkwijk, 2014) we report a highly-powered cross-sectional study of the association between maladaptive perfectionism and shame regulation strategies. In this discussion, we will focus on the clinical relevance of our results.

Our hypotheses were based upon the effect the shame regulation style might have on the intensity of the shame experience in association with intensity of experiences of maladaptive perfectionism: attacking the self and attacking others should sustain the emotional intensity of the shame and maladaptive experience, whereas withdrawal and avoidance should mitigate the intensity (Elison et al., 2006a). Although the expected large effect size was not found, our first hypothesis was supported: we found a medium positive association between maladaptive perfectionism and attacking the self. It confirms that this regulation style sustains the intensity of the shame and perfectionism discrepancy. Quite interestingly, Yelsma et al. (2002) found a very similar effect size $(\beta=0.46)$ for the association between Attack Self and self-esteem. Naturally, self-esteem and perfectionism discrepancy share variance, but these findings could be further studied to elucidate whether self-esteem mediates or moderates the association between attacking the self and maladaptive perfectionism. One step further, it would be of great importance to elucidate these relationships in light of depression severity (Rice et al., 1998) or therapeutic progress (Schalkwijk, 2014).

Secondly, we hypothesized a weak negative association between maladaptive perfectionism and withdrawal as the shame intensity is diminished, yet a weak positive 
association was observed. Withdrawing from potentially shameful situations may be quite a conscious act: withdrawing is still a confirmation of the self as vulnerable (Riggs et al., 2002) which is the same psychodynamic mechanism as experiencing a discrepancy between the goal and self-evaluation.

Furthermore, avoidance tries to minimize the experience of shame by not paying too much attention to it, or even denying it (Elison et al., 2006a). We hypothesized a weak positive association between maladaptive perfectionism and avoidance, but the association was non-existent under the selection constraints of our model. Given the study's high power, we are confident that in the case the model was false in rejecting the association between maladaptive perfectionism and avoidance, the association would have been negligible in effect size. Lastly, we hypothesized that maladaptive perfectionism would be strongly negatively associated with regulating shame by attacking others, but no significant association was found.

Understanding the relation between shame regulation styles and maladaptive perfectionism by looking at the change in intensity of the experience was not possible, but it proved to be fruitful to interpret the data in the frame of the diagnostic difference between internalizing and externalizing personality styles. In the DSM-5, personality disorders are grouped into internalizing disorders with prominent anxiety, depressive and somatic symptoms and externalizing disorders with prominent impulsive, disruptive conduct, and substance use symptoms. In the same way, attacking the self and withdrawing are internalizing ways of shame regulation as the source of the shame is attributed to oneself, whereas avoiding and attacking others are externalizing strategies, as the source of shame is placed outside oneself or its existence blandly denied (Schalkwijk et al., 2016). Our results now make important clinical sense: Subjects with maladaptive perfectionism, that is, subjects who perceive the gap between their standards and their performance as a personal failure, also attribute the source of their shame to themselves. They exhibit criticism in selfevaluation and tend to choose to withdraw from similar experiences. This explanation also fits with the interaction effect between attacking the self and withdrawal. With a clinically marginal effect size, the adherence of both regulation styles makes for a slightly larger discrepancy in perfectionism-goals and evaluation of the self. This could underwrite the argument of especially conscious withdrawal being associated with maladaptive perfectionism. The regulation style attacking the self acknowledges the experience of shame including awareness of one's negative actions (Elison et al., 2006a). If this also results in the conscious decision of further withdrawal from similar situations, the discrepancy of 
perfectionism goals and self-evaluation increases even further. Similarly, Yelsma et al. (2002) found that self-esteem was associated with internalizing coping styles with similar effect sizes, but not with attack others nor avoiding.

\subsection{Clinical implications for treatment of maladaptive perfectionism}

The meta-analysis of Smith et al (2016) suggested that perfectionism dimensions are part of the premorbid personality of people vulnerable to depressive symptoms: Seven perfectionism dimensions contributed to perfectionism-depression effects in a small magnitude. Rice and Stuart (2010) found that maladaptive perfectionism had a strong positive association with a motivational style aiming at avoiding pain. At the same time, our research shows, they are experiencing the pain of shame. Clinically, this is especially so when in treatment the patterns of maladaptive perfectionism must come into the open: the client feels exposed and experiences being vulnerable due to his low self-esteem and, consistent with his shame regulation style, shame grows. And with shame mounting, defense mechanisms come into action, hindering therapeutic progress (Lichtenberg et al., 2011; Steiner, 2011) or weaking the therapeutic relationship (Black et al., 2013). Our research might account for the negative responsiveness of perfectionism to brief therapies for depression (Blatt et al., 1998): there is not enough focus on and time for working through the evoked shame. Also in the realm of depression, the presumed interaction between maladaptive perfectionism, self-esteem and shame might be an important focus in the treatment as factors contributing to its etiology and maintenance.

\subsection{Limitations}

Although we claim that the results are clinically relevant for treatment, the research was done in a non-clinical setting and further research is needed addressing the proposed research questions in a clinical population. A further drawback of our results is that our data information set only contained the Discrepancy score of the APS-R, which blocked out the possibility of going into adaptive perfectionism. A replication of our study including the High Standards and Order subscales might further differentiate the clinical picture. Lastly, the cross-sectional nature of this study makes it impossible to infer causal relationships, nor can't 
the discussed associations be interpreted at the individual level.

Notwithstanding these limitations, we have shown that the associations between maladaptive perfectionism and shame regulating strategies are of paramount importance for therapeutic strategies in psychotherapeutic practice. 


\section{References}

Ashby, J.S., Rice, K.G., Martin, J.L., 2006. Perfectionism, shame, and depressive symptoms. J. Couns. Dev. 84, 148-156.

Benjamins, J.S., Migliorati, F., Dekker, K., Wassing, R., Moens, S., Blanken, T.F., te Lindert, B.H.W., Sjauw Mook, J., Van Someren, E.J.W., 2016. Insomnia heterogeneity: characteristics to consider for data-driven multivariate subtyping. Sleep Med. Rev., accepted.

Black, R.S., Curran, D., Dyer, K.F., 2013. The impact of shame on the therapeutic alliance and intimate relationships. J. Clin. Psychol. 69, 646-654.

Blatt, S.J., Zuroff, D.C., Bondi, C.M., Sanislow, C.A., Pilkonis, P.A., 1998. When and how perfectionism impedes the brief treatment of depression: Further analyses of the national institute of mental health treatment of depression collaborative research program. J. Consult. Clin. Psychol. 66, 423-428.

Cohen, J. (1992). A power primer. Psychological Bulletin, 112, 155-159.

Egan, S.J., Wade, T.D., Shafran, R., 2011. Perfectionism as a transdiagnostic process: A clinical review. Clin. Psychol. Rev. 31, 203-212.

Elison, J., Lennon, R., Pulos, S., 2006a. Investigating the compass of shame: The development of the compass of shame scale. Soc. Behav. Pers. 34, 221-238.

Elison, J., Pulos, S., Lennon, R., 2006b. Shame-focused coping: An empirical study of the compass of shame. Soc. Behav. Pers. 34, 161-168.

Fedewa, B.A., Burns, L.R., Gomez, A.A., 2005. Positive and negative perfectionism and the shame/guilt distinction: adaptive and maladaptive characteristics. Pers. Individ. Dif. $38,1609-1619$

Frost, R.O., Marten, P., Lahart, C., Rosenblate, R., 1990. The Dimensions of Perfectionism. Cognit. Ther. Res. 14, 449-468.

Glickauf-Hughes, C., Wells, M., 1995. Narcissistic characters with obsessive features: diagnostic and treatment considerations. Am. J. Psychoanal. 55, 129-143.

Hewitt, P.L., Flett, G.L., 1991. Perfectionism in the self and social contexts: conceptualization, assessment, and association with psychopathology. J. Pers. Soc. Psychol. 60, 456-470.

Jahromi, F.G., Naziri, G., Barzegar, M., 2012. The relationship between socially prescribed perfectionism and depression: The mediating role of maladaptive cognitive schemas. 4th International Conference of Cognitive Science 32, 141-147.

Kim, L.E., Chen, L., MacCann, C., Karlov, L. \& Kleitman, S. (2015). Evidence for three 
factors of perfectionism: Perfectionistic Strivings, Order, and Perfectionistic Concerns. Personality and Individual Differences, 84, 16-22.

Lichtenberg, J.D., Lachmann, F.M., Fosshage, J.L., 2011. Psychoanalysis and motivational systems: A new look. Routledge, New York, NY, USA

Malinowski, A.S., Veselka, L. \& Atkinson, B.E. (2017). An investigation of vulnerability factors for depression. Personality and Individual Differences, 107, 126 - 130.

Mann, M.P., 2004. The adverse influence of narcissistic injury and perfectionism on college students' institutional attachment. Pers. Individ. Dif. 36, 1797-1806.

Mills, R.S.L., 2005. Taking stock of the developmental literature on shame. Dev. Rev. 25, 26-63.

Rice, K.G., Ashby, J.S., Slaney, R.B., 1998. Self-esteem as a mediator between perfectionism and depression: A structural equations analysis. J. Couns. Psychol. 45, 304-314.

Rice, K.G., Ashby, J.S., Slaney, R.B., 2007. Perfectionism and the five-factor model of personality. Assessment 14, 385-398.

Rice, K.G., Stuart, J., 2010. Differentiating adaptive and maladaptive perfectionism on the MMPI-2 and MIPS revised. J. Pers. Assess. 92, 158-167.

Riggs, S.A., Jacobvitz, D., Hazen, N., 2002. Adult, attachment and history of psychotherapy in a normative sample. Psychotherapy 39, 344-353.

Schalkwijk, F., 2014. Shame in Psychotherapy. EFPP Psychoanalytic Psychotherapy Review, $2-9$.

Schalkwijk, F., 2015. The conscience and self-conscious emotions in adolescence: An integrative approach, 1st ed. Routledge, New York, NY, USA

Schalkwijk, F., Stams, G.J., Dekker, J., J, P., Elison, J., (2016). Measuring shame coping: The validation of the Compass of Shame Scale. Journal of Social Behavior and Personality 44, 1775 - 1792

Schalkwijk, F., Stams, G.J., Stegge, H., Dekker, J., Peen, J., 2016. The Conscience as a Regulatory Function: Empathy, Shame, Pride, Guilt, and Moral Orientation in Delinquent Adolescents. Int. J. Offender Ther. Comp. Criminol. 60, 675-693.

Sironic, A., Reeve, R.A., 2015. A Combined Analysis of the Frost Multidimensional Perfectionism Scale (FMPS), Child and Adolescent Perfectionism Scale (CAPS), and Almost Perfect Scale-Revised (APS-R): Different Perfectionist Profiles in Adolescent High School Students. Psychol. Assess. 27, 1471-1483.

Slaney, R.B., Rice, K.G., Ashby, J.S., 2002. A programmatic approach to measuring perfectionism: The Almost Perfect Scales, In: Flett, G.L., Hewitt, P.L. (Eds.), 
Perfectionism: Theory, research, and treatment, 1st ed. American Psychological Association, Washington, DC, USA, p. 435.

Slaney, R.B., Rice, K.G., Ashby, J.S., 2002. A programmatic approach to measuring perfectionism: The Almost Perfect Scales, In: Flett, G.L., Hewitt, P.L. (Eds.), Perfectionism: Theory, research, and treatment, 1st ed. American Psychological Association, Washington, DC, USA, p. 435.

Smith, M.M., Saklofske, D.H., Yan, G. \& Sherry, S.B. (2017). Does perfectionism predict depression, anxiety, stress, and life satisfaction after controlling for neuroticism? Journal of Individual Differences, 38, 63-70.

Smith, M.M., Sherry, S.B., Rnic, K., Saklofske, D.H., Enns, M. \& Gralnick, T. (2016). Are perfectionism dimensions vulnerability factors for depressive symptoms after controlling for neuroticism? A meta-analysis of 10 longitudinal studies. European Journal of Personality, 30, 201-212.

Steiner, J., 2011. Seeing and being seen: Emerging from a psychic retreat, 1st ed. Routledge, New York, NY, USA

Stoeber, J., Harris, R.A., Moon, P.S., 2007. Perfectionism and the experience of pride, shame, and guilt: Comparing healthy perfectionists, unhealthy perfectionists, and nonperfectionists. Personality and Individual Differences, 43, 131-141.

Stoeber, J., Kobori, O., Tanno, Y., 2013. Perfectionism and Self conscious Emotions in British and Japanese Students: Predicting Pride and Embarrassment after Success and Failure. European Journal of Persoanlity, 27, 59-70.

Stoeber, J. \& Otto, K. (2006). Positive conceptions of perfectionism: Approaches, evidence, challenges. Personality and Social Psychology Review, 10, 295-319.

Stoeber, J., Yang, H.F., 2010. Perfectionism and emotional reactions to perfect and flawed achievements: Satisfaction and pride only when perfect. Personality and Individual Differences, 49, 246-251.

Tangney, J.P., Dearing, R.L., 2003. Shame and guilt, 1st ed. Guilford Press, New York, NY, USA

Yelsma, P., Brown, N.M., Elison, J., 2002. Shame-focused coping styles and their associations with self-esteem. Psychol. Rep. 90, 1179-1189. 